\title{
Short Term Electric Load Forecasting using Neural Network and Genetic Algorithm
}

\author{
Mahrufat D. Olagoke \\ Computer Science \\ Department, \\ University of llorin, \\ Ilorin-Kwara State
}

\author{
A.A. Ayeni, PhD \\ Telecommunication Science \\ Department, \\ University of Ilorin, \\ Ilorin-Kwara State
}

\author{
Moshood A. Hambali \\ Computer Science \\ Department, \\ Federal University Wukari, \\ Wukari-Taraba State
}

\begin{abstract}
The major predicament with electricity as a means of transporting energy is that it cannot be stored unlike gas, oil, coal or hydrogen. Due to this, the electric power company faces economical and technical problems in planning, operation and control of electric power system. For the purpose of optimal planning and operation of an electric power system, there is need for appropriate evaluation of the present and future electric load. Electric load forecasting is used by electric power company to anticipate the amount of energy needed to meet up with the demand. Various statistical and artificial intelligence techniques have been applied to short term electric load forecasting in the past but were hampered with some drawbacks. This paper presents another approach for short term load forecasting with lead time of a day ahead (1-24 hours) using artificial neural network (ANN). The hidden layer in ANN model was generated using genetic algorithm instead of the usual practice of trial and error; the ANN model was trained by Levenberg Marquardt. The data (daily load data of 330/132/33KV substation, Ganmo, Kwara State for the month of May, 2014) used in training and validation of the neural network was obtained from the Transmission Company of Nigeria, National Control Centre, Osogbo, Osun State, Nigeria. The model for short term load forecast was designed and implemented with MATLAB package. The result was evaluated by Mean Absolute Percentage Error (MAPE) of 4.705 for the forecasted day.
\end{abstract}

\section{General Terms}

Short Term Electric Load Forecasting, Generic Algorithm, Artificial Neural Network

\section{Keywords}

Short Term Electric Load Forecasting, Artificial Neural Network, Genetic Algorithm and Levenberg Marquardt

\section{INTRODUCTION}

The key function of an electric power company is to supply customers with high quality electric energy in secured and economical manner. In order to do so, an electric power company faces economical and technical problems in planning, control and operation of electric power system. For the purpose of optimal planning and operation of electric power system, there is need for proper evaluation of present day and future electric power load $[1,2]$.

An electric load forecasting is used by an electric power company to anticipate the amount of electric energy needed to supply so as to meet up the demand. Electric load forecasts can be divided into three categories based on the planning perspective of the duration: Short Term Load Forecasts
(STLF): This is usually from one hour to one week. Medium Term Load Forecasts (MTLF): This is usually from one week to a month. Long Term Load Forecasts (LTLF): This is longer than a year.

The forecasts for different time horizons are important for different operations with an electric power company. The natures of these forecasts are different as well. For instance, for a particular region, it is possible to predict the next day load with an accuracy of approximately $1-3 \%$. However, it is impossible to predict the next year peak load with similar accuracy, since accurate long-term weather forecasts are not available [3]. STLF can help electric load planners to estimate load flows and make decisions that prevent overloading. Timely implementations of such decision lead to the improvement of network reliability and reduce occurrences of equipment failures and blackouts. MTLF is useful in unit maintenance and determining the quantity of fuel to purchase in power plants. LTLF used to supply electric power company management with prediction of future needs for expansion, equipment purchases and inter-tie tariff setting [3].

For STLF, several factors should be considered such as time factor, weather data and possible customer's classes. The time factors include the time of the year, the day of the week and the hour of the day. There are significant differences in load between weekdays and weekends. The load on different weekdays also can behave differently. For example, Mondays and Fridays being adjacent to weekends may have structurally different loads than Tuesday through Thursday. This is particularly true during the summer time. Holidays are more difficult to forecast than non-holidays because of their relative infrequent occurrence. Weather conditions influence the load. In fact, forecasted weather parameters are the most important factors in STLF. Various weather variables could be considered for load forecasting. Temperature and humidity are the most commonly used load predictors. Most electric utilities serve customers of different types such as residential, commercial and industrial. The electric usage pattern is different for customers that belong to different classes but is somewhat alike for customers within each class. Therefore, most utilities distinguish load behaviours on a class-by-class basis [3].

Many methods have been employed for load forecasting in the past; they are based on various statistical methods such as time series, regression, Box-Jenkins model, exponential smoothing and so on. However, the statistical models provide physical transparency in interpretation of data and reasonable accuracy in STLF but with the associated problems, such as limited modeling and heavy computational effort make them less preferable over intelligent techniques [2]. 
In recent years, research has converged towards methods that use artificial intelligence (AI) such as fuzzy logic, artificial neural networks and expert system. Among the AI methods, artificial neural networks (ANN) have received a great deal of attention by researchers in this area of research due to its flexibility in data modeling [2].

ANN is a mathematical model or computational model based on biological neural system. It consists of an interconnected group of artificial neurons and processes information using a connectionist approach to computation. ANN has been particularly successful in predicting the behaviour of large complex and nonlinear systems with a large number of variables [4]. An ANN model takes a set of inputs and produces one or more output and does not require any preassumptions on logical or analytical form between the input and output variables. Its mapping capability is obtained through the architecture of the network and training of its parameters with experimental data by examining the patterns between the inputs and their corresponding outputs. A neural network gains the knowledge to predict a system's output for any given inputs. These features make a neural network an ideal model for predicting the behaviour of a complex engineering system.

However, their application to some real world problems has been hampered by their long training time; there is no clear guideline for determining the number of hidden nodes in hidden layer(s) etc., it is the usual practice to use trial and error to find suitable neural network architecture for a given problem. This method is not only time consuming but may not generate optimal neural network architecture. One of the methods to overcome these difficulties in designing ANN is by using genetic algorithm (GA) [5, 6]. GA is a global search algorithm which operates on a population of rules. Based on the mechanics of selection and natural genetics, it promotes over time the rules that perform well in a given environment and introduces into the population new rules to be tried. Rules are coded as binary strings of finite length. The measure of the rules' performance is defined by their fitness function.

The aim of this research work is to develop an ANN model for short term electric load forecasting with lead time of one day ahead using genetic algorithm to design hidden nodes of ANN model.

\section{STATEMENT OF THE PROBLEM}

An electric power company faces economical and technical challenges in planning, control and operation of an electric power system, for the purpose of optimal planning and operation of an electric power system, there is need for proper evaluation of present day and future electric power load [1, 2].Various methods have been proposed to solve the demanding task of STLF, but are associated with some drawbacks. This paper therefore, proposes a new method for STLF using ANN and GA.

\section{LITERATURE REVIEW}

For years the problems of improving the accuracy of load forecasting has been an important topic of research. Research in this area in the last years has resulted in the development of numerous forecasting methods [7]. These methods are mainly classified into two categories: Classical approaches and artificial intelligence (AI) based techniques. Classical approaches are based on various statistical modeling methods such as time- series, regression, exponential smoothing, BoxJenkins model and Kalman filters. However, these classical methods cannot properly represent the complex non-linear relationships that exist between the load and series of factors that influence it [1].

As from 1990s, researchers began to use different approaches for STLF other than classical approach. The emphasis shifted to the implementation of various AI techniques for STLF. AI techniques such as neural network, fuzzy logic and expert systems have been applied to deal with the non-linearity, large data sets requirement in implementing the STLF system and other difficulties in modeling of classical methods used for the application of STLF [1]. Among the AI techniques available, different models of neural network have received a great deal of attention by researchers in area of STLF due to its flexibility in data modeling. Park [8] were among the first group of researchers who used the ANN approach for STLF.

Srinvasan, Liew and Chen, [9] used an ANN based on back propagation for forecasting, and showed its superiority to traditional methods. Chen, Tzeng, and Hwang, [10] applied a supervisory functional ANN technique to forecast for three substations in Taiwan.

Christopher and Francis, [11] developed a supervised ANN based model for STLF and evaluated the performance of the model by applying the actual load data of the Kenya National grid power system.

Amera, Lamya and Sozan, [12] analyzed and discussed a comprehensive approach for STLF using ANN. Their proposed architectures were trained and tested using previous two years actual load data obtained from Duhuk ELC control in Iraq. In their study, four ANN models were implemented and validated with reasonable accuracy on real electric load generation output data.

Samsher and Unde, [1] carried out STLF for P.D.V.V.P COE, Ahmednager college campus using ANN technique and was implemented on MATLAB-10.

Adepoju, Ogunjuyigbe and Alawode, [13] used a multi-layer perceptron (MLP) networks with back propagation to adjust the weights of the neural network. The neural network was trained using data from the Nigeria power company.

Mohammed and Sanusi, [2] developed a multilayer feed forward ANN model for $132 / 33 \mathrm{KV}$ substation, Kano, Nigeria using Levenberg- Marquardt optimization technique to train the network.

Slobodan et al, [14] presented a method for STLF, based on ANN targeted for used in large-scale system as distribution management system (DSM). The system comprises of a preprocessing unit (PPU) and a feed forward ANN ordered in a sequence. Functionality of the proposed method was tested on recorded data from Serbian electrical utility.

ANNs have been integrated with several other techniques to improve their accuracy. Nima and Farshid, [15] presented a hybridization of a neural network with a novel stochastic search technique for STLF.

Zohreh, hadi, and Mahdi, [16] used adaptive neural-fuzzy inference system (ANFIS) to study the design of STLF systems for the east of Iran.

Chaturvedi and Sinha, [17] Presented the design of STLF system for $33 \mathrm{KV}$ substation of Dayalbagh Educational Institute, Agra using Neural-wavelet based hybrid model. 
This research work therefore, proposes a new method for STLF using ANN and genetic algorithm. The hidden layer of neural network will be designed using GA and trained by Levenberg Marquardt.

\section{METHODOLOGY}

\subsection{Data Collection and Preparation}

The historical data (load data and temperature) were collected for this research. The historical load data was obtained from the transmission company of Nigeria, National control centre, Osogbo, Osun State, Nigeria. And the temperature was obtained from the internet. The data collected is the actual load data of the 330/132/33 KV substation, Ganmo, Kwara State for the Month of May, 2014. The first twenty one days load data and temperature will be used for training the network and the load data and temperature for the remaining days in the Month will be used for the network validation.

Table 1 shows the sample of a day load dataset used for training the network:

Table 1. Sample of a Day Load Dataset

\begin{tabular}{|c|c|c|c|}
\hline Date & Hrs & Load & Temp \\
\hline $1 / 5 / 2014$ & 1 & 25.000 & 28.50 \\
\hline $1 / 5 / 2014$ & 2 & 13.000 & 28.50 \\
\hline $1 / 5 / 2014$ & 3 & 0.000 & 28.50 \\
\hline $1 / 5 / 2014$ & 4 & 0.000 & 28.50 \\
\hline $1 / 5 / 2014$ & 5 & 0.000 & 28.50 \\
\hline $1 / 5 / 2014$ & 6 & 0.000 & 28.50 \\
\hline $1 / 5 / 2014$ & 7 & 0.000 & 28.50 \\
\hline $1 / 5 / 2014$ & 8 & 0.000 & 28.50 \\
\hline $1 / 5 / 2014$ & 9 & 17.400 & 28.50 \\
\hline $1 / 5 / 2014$ & 10 & 56.000 & 28.50 \\
\hline $1 / 5 / 2014$ & 11 & 57.000 & 28.50 \\
\hline $1 / 5 / 2014$ & 12 & 24.000 & 28.50 \\
\hline $1 / 5 / 2014$ & 13 & 31.000 & 28.50 \\
\hline $1 / 5 / 2014$ & 14 & 31.000 & 28.50 \\
\hline $1 / 5 / 2014$ & 15 & 30.000 & 28.50 \\
\hline $1 / 5 / 2014$ & 16 & 40.000 & 28.50 \\
\hline $1 / 5 / 2014$ & 17 & 45.000 & 28.50 \\
\hline $1 / 5 / 2014$ & 18 & 45.000 & 28.50 \\
\hline $1 / 5 / 2014$ & 19 & 18.000 & 28.50 \\
\hline $1 / 5 / 2014$ & 20 & 0.000 & 28.50 \\
\hline $1 / 5 / 2014$ & 21 & 57.000 & 28.50 \\
\hline $1 / 5 / 2014$ & 22 & 53.000 & 28.50 \\
\hline $1 / 5 / 2014$ & 23 & 62.000 & 28.50 \\
\hline $1 / 5 / 2014$ & 24 & 59.000 & 28.50 \\
\hline
\end{tabular}

\subsection{Data Preprocessing}

Scaling of raw input data is usually necessary to reduce the bias caused by different measuring unit of original input variables. The approach used for scaling the network input and target was to normalize the mean and standard deviation of the training set.

\subsection{Network Structure Design}

The next step after splitting the training and validation data set is to design the structure for neural network. This has to do with selecting network topology and determination of the input nodes, output nodes, number of hidden layers and number of hidden nodes. Network topology is usually determined based on the type of task to be performed by the network proposed. Multilayer feed forward neural network has been successfully applied for prediction (regression and classification) problems [4]. Multilayer feed forward will be used in this research. The number of input nodes is usually set equal to the number of input variables.

The following are the input variables for this research

(i) Hour of the day

(ii) Temperature (average temperature)

(iii) Day of the week

The output of the neural network represents the forecasted load data for the forecasting day. Determining the number of hidden layer(s) and number of nodes in the hidden layer(s) is a critical decision in the design of neural networks. Too many hidden neurons lead to many trainable weights, which can make a neural network to become erratic and unreliable. On the other hand too few hidden neurons restrict the learning ability of a neural network and deteriorate its approximation performance [18]. However, there is no clear guideline for determining the number of neurons in hidden layer(s). The usual practice is by using trial and error which may not yield an optimal network design and the process is time consuming. One of the ways of overcoming these difficulties in designing ANN is to use GA $[19,6]$

Using GA to design ANN encompasses two major attracting features. First, it automates the design of the network which would have done manually. Second, the design process is analogous to a biological process in which the neural network blueprints are encoded in chromosome. The following are the GA steps for selecting the optimum topology for the neural network model.

1. Set the initial population (neural network parameters are represented by the chromosome).

2. Evaluate the fitness function for each individual in the population.

3. Select the first two individuals with highest fitness value in the current generation and apply cross over and mutation operators.

4. Repeat from step 2 until all individuals in the population met the convergence criteria.

Decode the converged individuals in the final generation and obtain the optimized neural network structure with optimal number of input neurons and hidden layer neurons.

\subsection{Network Training}

After the network has been designed, the next step is to train the network. Training of an artificial neural is an iterative process that has to do with adjusting of the connection weight. Back propagation algorithm has been widely used in the past as a basic learning algorithm for training feed-forward neural networks, but takes a very long time in training due to the 
nature of gradient descent [20]. Several techniques have been used to improve the performance of back propagation, among them is by incorporating Levenberg Marquardt. Levenberg Marquardt will be adopted for training neural network in this research. Levenberg Marquardt is numerical optimization based technique.

For Lenvenberg-Marquardt optimization the performance index to be optimized is defined as:

$$
\mathrm{F}(\mathrm{w})=\sum_{p}^{p=1}\left[\sum_{k=1}^{k}(d k p-O k p) 2\right]-----(1)
$$

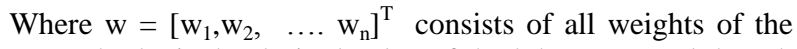
network, $d_{k p}$ is the desired value of the kth output and the pth pattern, $O_{k p}$ is the actual value of the kth output and the pth pattern, $\mathrm{N}$ is the number of the weights, $\mathrm{P}$ is the number of the patterns and $\mathrm{k}$ is the number of outputs. Levenberg Marquardt combines the speed of Gauss-newton's method and the stability of error back propagation algorithm during training steps, Levenberg Marquardt learning rule utilizes a scalar (combination coefficient) $\mu$ to control the shift between Gauss-netwon method and error back propagation algorithm during training steps (equation 2). When $\mu$ is large, the learning process approximate error back propagation algorithm. When $\mu$ is small, the learning process essentially follows the Gauss-netwon rule. The value of $\mu$ increases, if the error increases after an iteration step and decreases when a step reduces the error note that $\mathbf{J}$ is the jacobian matrix defined on error vector e.

$$
\triangle \mathrm{W}=\left(\mathrm{J}^{\mathrm{T}} \mathrm{J}+\mu \mathrm{I}\right)^{-1} \mathrm{~J}^{\mathrm{T}} \mathrm{e}
$$

The Jacobian matrix is defined as

$$
J=\left[\begin{array}{ccc}
\frac{\partial F\left(x_{1}, w\right)}{\partial w_{1}} & \cdots & \frac{\partial F\left(x_{1}, w\right)}{\partial w_{W}} \\
\vdots & \ddots & \vdots \\
\frac{\partial F\left(x_{N}, w\right)}{\partial w_{1}} & \cdots & \frac{\partial F\left(x_{N}, w\right)}{\partial w_{W}}
\end{array}\right]
$$

In neural network the jacobian matrix is a $\mathrm{N}$ - by- $\mathrm{W}$ matrix, where $\mathrm{N}$ is the number of entries in our training set and $\mathrm{W}$ is the total number of parameters (weights + biases) of the network.

\subsection{General Levenberg-Marquardt Algorithm}

Each learning iteration in Levenberg Marquardt contains the following basic steps

1. Compute the jacobian matrix

2. Compute the error gradient $g=J^{t} E$

3. Approximate the hessian using the cross product jacobian $\mathrm{H}=\mathrm{J}^{\mathrm{T}} \mathrm{J}$

4. Solve $(H+\lambda I) \delta=g$ to find $\delta$

5. Update the network weights w using $\delta$

6. Recalculate the sum of squared errors using updated weights

7. If the sum of the squared error has not decreased, discard the weights, increase $\lambda$ using $\mathrm{v}$ and go to step 4

8. Else decrease $\lambda$ using $\mathrm{v}$ and stop.

\subsection{Network Validation}

After the network has been properly trained, it needs to be validated on its performance of generalization. New data that are not used during training process are feed in to the network to see whether it can predict well at these "unseen" data

\section{RESULT AND DISCUSSION}

The data set is preprocessed before training and validation with function mapstd. This function allows the inputs and targets to have a mean of zero and standard deviation of unity.

\subsection{Training, Validation and Testing of the Network}

Neural network parameters used in the experiment, is summarized in table 2.

\section{Table 2: Summary of Network Parameters}

Network topology: Multi-layer feed forward neural network

Input variables: day of the week, hour of the day and the average temperature

Number of hidden neuron: automated (using GA)

Number of epoch -91

Training algorithm - Levenberg -Marquardt (trainlm)

Transfer function used in the hidden layer - sigmoid function Transfer function used in the output layer - sigmoid function Sigmoid function is defines as:

$$
\mathrm{Y}_{\mathrm{T}}=1 /\left(1+\mathrm{e}^{-\mathrm{y}}\right)
$$

Where $\mathrm{Y}_{\mathrm{T}}$ is the transformed (i.e. normalized) value of $\mathrm{Y}$

\subsection{Results Analysis and Evaluation}

Performance plot (fig. 1) indicates the behaviour of the network during training, validation and testing. The performance goal was met at 91 epochs (number of iterations). This figure shows the mean absolute error (MAE) versus epochs; revealed that, mean absolute error was decreasing during the learning process. 


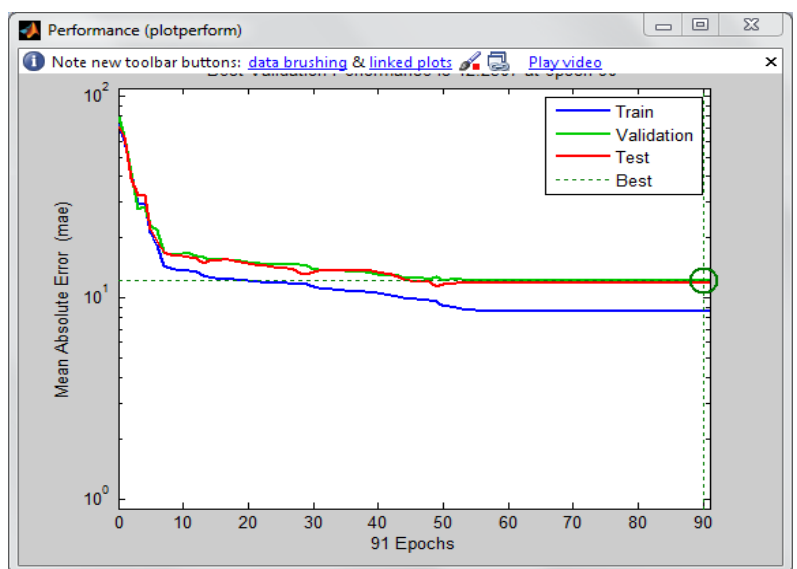

Fig. 1: Performance Plot

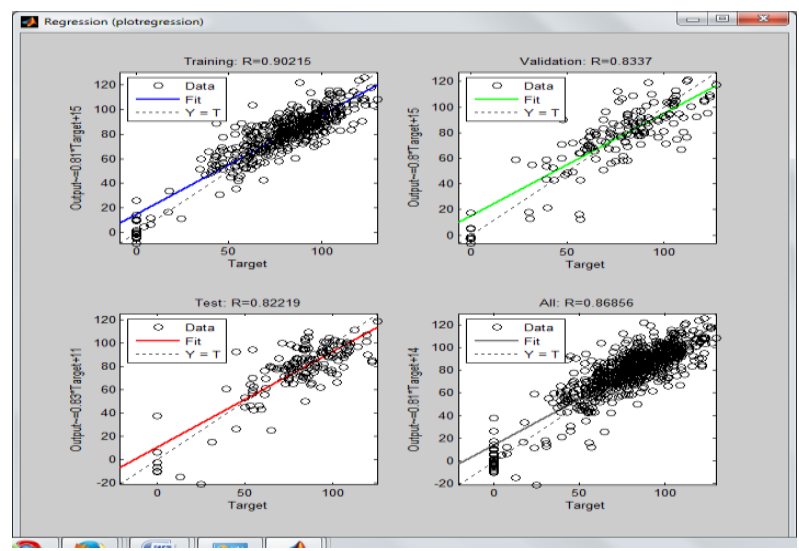

Fig. 2: Regression Plot

This graph (fig. 2) shows the closeness between the outputs and the target. A $R$ value of one $(R=1)$ indicates a perfect relation between the target and the output. From the regression plot $R=0.90215$ for training, $R=0.8337$ for validation, $R=0.82219$ for testing and $R=0.86856$ for all.

This shows that the target and output values are very close. This means that the network predicted the output satisfactorily.

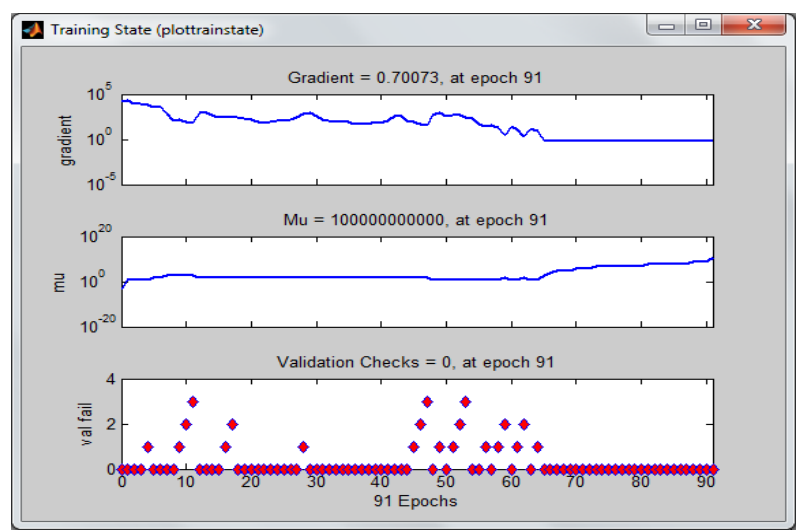

Fig. 3: Training State Plot

The figure 3 consists of three different graphs. The first plot is the learning function versus number of epochs. This shows the trend of the gradient values as the number of computational iterations increases. It is necessary in monitoring the manner in which the training progresses. The second plot is that of the learning rate $(\mathrm{mu})$ against increasing number of epochs. This plot is essential in monitoring the rate at which the computed network error reduces during the progress of the training. The final plot here is that of the validation checks carried out automatically any time a sudden change is observed in the network gradient computation is carried out.

Figure 4 shows the comparison between actual load data obtained from the power company which is very close to the results obtained from the trained neural network model output data.

\subsection{Result Evaluation}

Mean absolute percentage error (MAPE) is used in the evaluation of the results and is defines as:

$$
\text { MAPE }=\left(\frac{\left|\underline{x}_{i}-y_{i}\right|}{x_{i}}\right) \times 100
$$

Where $x_{i}$ represent the actual values and $y_{i}$ represent the predicted values.

The MAPE for the forecasting day is $4.705 \%$ and the MAPE for the historical load is $4.88 \%$

\subsection{Output Data}

The hourly forecast for the next day with average temperature of $30^{\circ} \mathrm{C}$. 


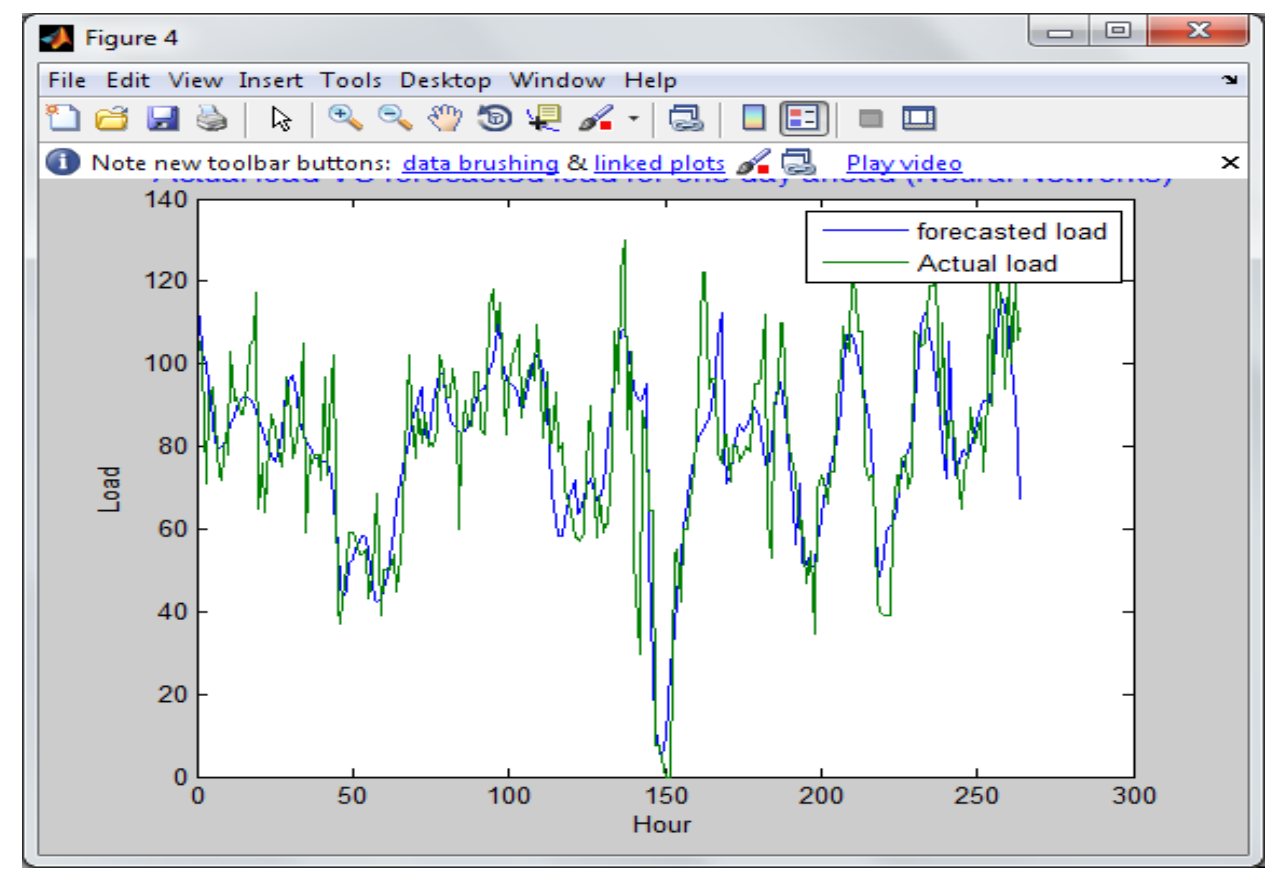

Fig. 4: Comparison of forecasted load and Actual load

Table 3: Forecasted Load for a Day

\begin{tabular}{|l|l|l|l|}
\hline HOURS & $\begin{array}{l}\text { PREDICTED } \\
\text { LOAD }\end{array}$ & HOURS & $\begin{array}{l}\text { PREDICTED } \\
\text { LOAD }\end{array}$ \\
\hline 1 & 49.4 MWatts & 2 & 55.2 MWatts \\
\hline 3 & 60.3 MWatts & 4 & 64.2 MWatts \\
\hline 5 & 65.9 MWatts & 6 & 63.9 MWatts \\
\hline 7 & 56.8 MWatts & 8 & 47.6 MWatts \\
\hline 9 & 41.8 MWatts & 10 & 41.4 MWatts \\
\hline 11 & 47.3 MWatts & 12 & 58.7 MWatts \\
\hline 13 & 65.0 MWatts & 14 & 64.4 MWatts \\
\hline 15 & 58.7 MWatts & 16 & 50.0 MWatts \\
\hline 17 & 39.7 MWatts & 18 & 28.9 MWatts \\
\hline 19 & 18.1 MWatts & 20 & 7.7 MWatts \\
\hline 21 & 2.1 MWatts & 22 & 11.3 MWatts \\
\hline 23 & 19.7 MWatts & 24 & 27.6 MWatts \\
\hline
\end{tabular}

\subsection{Contribution to Knowledge}

STLF play a vital role in planning and operation of electric power system, it can help an electric load planners to estimate load flows and to make decisions that can be prevent overloading. Timely implementations of such decisions can lead to the improvement of network reliability and reduces occurrence of equipment failures and blackout.

\section{CONCLUSION}

A new method for short term electric load forecasting was developed using ANN optimized with GA. The use of GA to design ANN encompasses two major attracting features. Firstly, it automates the design of the network which would have been done manually. Secondly, the design process is analogous to a biological process in which the neural network blueprints are encoded in chromosomes. The training, validation and testing of the ANN was conducted offline using MATLAB. The results obtained in this work confirm the efficiency of ANN in short term electric load forecasting. The model was able to determine the non-linear relationship that exists between the historical load data and temperature. With forecasted average temperature for the forecasting day, the model could make a prediction of the next day (hourly) load.

\section{FUTURE WORK}

For future works, the error in the network can be further reduced if a larger set of data is used for the network training. Also, additional information such as customer class can be included in the network so as to obtain a more representative forecast of future load.

\section{REFERENCES}

[1] Samsher Kadir Shiekh and Unde M. G. 2012. Short Term Load Forecasting Using ANN Technique. International Journal of Engineering Sciences and Emerging Technologies Vol 1.Issue 2, Pp: 99-107.

[2] Mohammed Buhari and Sanusi Sani Adamu 2012. ShortTerm Load Forecasting Using ANN. Proceedings of the International Multi-conference of Engineers and Computer Scientist IMECS 2012, Hong Kong Vol. 1 Pg. 978-988.

[3] Eugene A. Feinberg and Dora Genethliou 2006. Book: Title / Chapter Applied Mathematics for Power Systems. "Chapter 12 Load Forecasting" Weather (2006), Issue: August, Publisher: Springer, Pg 269 -285

[4] Zhao H., Ren Z. and Huang W. 1997. Short Term Forecasting Considering Weekly Period Based on Periodical Auto Regression. Proceedings of the Chiness Society of Electrical Engineers.

[5] Geoffrey F. Miller, Peter M. Todd and Shailesh U. 
Hegde 1989. Designing Neural Networks Using Genetic Algorithms. Proceedings of the Third International Conference on Genetic Algorithms, Edited by J. David Schaffer Pg. 379-384.

[6] Belal Al- Khateeb and Maha Mahmood 2013. A Framework for an Automatic Generation of Neural Networks. International Journal of Computer Science Issues. Vol. 10 issue $3 \mathrm{Pg}$. 59-62.

[7] Gwo-Chung Liao and Ta- Peng Tsao 2006. Application of a Fuzzy Neural Network Combined With Chaos Genetic Algorithm and Simulated Annealing to Short Term Load Forecasting. Evolutionary Computation IEEE Transaction Vol. 10(3) Pg 330-340.

[8] Park, D. C., El- Sharkawi, M. A., Marks, R. A. II, Atlas, L. E. and Danborg, M. J. 1991. Electric Load Forecasting Using an Artificial Neural Network. IEEE Transactions of Power Engineering Vol. 6 Pg. 442-449.

[9] Srinvasan D., Liew A. C. and Chen S. P. 1991. A Novel Approach to Electric Load Forecasting Based on Neural Networks IEEE International Joint Conference of Neural Networks, Singapore.

[10] Christopher A. Moturi and Francis K. Kioko 2013. Use of Artificial Neural Networks for Short Term Electricity Load Forecasting of Kenya National Grid Power System. International Journal of Computer Applications Volume 63 Number 2 Pg 25-30.

[11] Chen, C. S, Tzeng, T. M and Hwang, J. C. 1996. Application of Artificial Neural Networks to Substation Load Forecasting. Electric Power Systems Research, 38,153-160.

[12] Amera Ismail Melhum, Lamya Abdullateef Omar and Sozan Addulla Mahmood 2013. Short Term Load Forecasting Using Artificial Neural Network.
International Journal of Soft Computing and Engineering (IJSCE), Vol. 3, Pg. 56-58.

[13] Adepoju, G. A, Ogunjuyigbe, S. O. A and Alawode, K. O. 2007. Application of Neural network to Load Forecasting in Nigerian Electrical Power System. The Pacific Journal of Science and Technology. Vol. 8 Number 1. Pg 68-72.

[14] Slobodan Ilic, Aleksandar Selakov, Srdan Vukmirovic, Aleksandar Erdeljan and Filip Kulic 2013. Short Term Load Forecasting in Large Scale Electrical Utility Using Artificial Neural Network, Journal of Scientific and Industrial Research Vol. 72, Pg 739-745.

[15] Nima Amjady and Farshid Keynia 2011. A New Neural Network Approach to Short Term Load Forecasting of Electrical Power Systems. Energies ISSN 1996-1073.

[16] Zohreh Souzanchi k, hadi Fanace T, Mahdi Yagbobi 2010. A Multi Adaptive Neuro Fuzzy Inference System For STLF by Using Previous Day Features. International Conference on Electronics and Information Engineering.

[17] Chaturvedi D.K and Premdayal S. A. 2013. NeuralWavelet Based Model for Short Term Load Forecasting. Control Theory and Informatics. Vol. 3 No. 2 Pg. 42-45.

[18] Vladimir, C and Mulier, F. 1998. Learning from Data: Concepts, Theory and Methods, John Wiley \& Sons. Inc, New York.

[19] Goldberg, D.E. 1989. Genetic Algorithms in Search, Optimization and Machine. Learning, Addison-Wesley, Reading, Massachusetts.

[20] Demith H. and beale M. (1998). Neural Network Toolbox, for Use with MATLAB, the Math Works www.mathworks.com 